Meta

Journal des traducteurs

Translators' Journal

\title{
Liste des relecteurs 2013
}

Volume 58, numéro 3, décembre 2013

URI : https://id.erudit.org/iderudit/1025044ar

DOI : https://doi.org/10.7202/1025044ar

Aller au sommaire du numéro

Éditeur(s)

Les Presses de l’Université de Montréal

ISSN

0026-0452 (imprimé)

1492-1421 (numérique)

Découvrir la revue

Citer ce document

(2013). Liste des relecteurs 2013. Meta, 58(3), 479-481.

https://doi.org/10.7202/1025044ar

Ce document est protégé par la loi sur le droit d'auteur. L'utilisation des services d'Érudit (y compris la reproduction) est assujettie à sa politique d'utilisation que vous pouvez consulter en ligne.

https://apropos.erudit.org/fr/usagers/politique-dutilisation/
Cet article est diffusé et préservé par Érudit.

Érudit est un consortium interuniversitaire sans but lucratif composé de l’Université de Montréal, l'Université Laval et l'Université du Québec à Montréal. Il a pour mission la promotion et la valorisation de la recherche. https://www.erudit.org/fr/ 


\section{Liste des relecteurs 2013}

Nous remercions chaleureusement tous ceux et celles qui ont donné de leur temps et qui ont contribué à la revue. Toute omission est involontaire de notre part, veuillez nous la signaler.

Mercè Altimir Losada (Universitat Autònoma de Barcelona)

Maïte Aragonés Lumeras (Organisation Mondiale de la Propriété Intellectuelle)

Pierre Auger (Université Laval)

Stefan Baumgarten (Bangor University)

Allison Beeby (Universitat Autònoma de Barcelona)

Henri Béjoint (Université Lumière Lyon 2)

Marie-Alice Belle (Université de Montréal)

Sanaa Benmessaoud (Université de Montréal)

Joyce Boro (Université de Montréal)

Lynne Bowker (Université d'Ottawa)

Annie Brisset (Université d'Ottawa)

Helena Buffery (University College Cork)

Joost Buysschaert (Hogeschool Gent)

Antoine Chalvin (Institut national des langues et civilisations orientales)

Po Suen Maria Cheng (City University of Hong Kong)

Jungwha Choi (Hankuk University of Foreign Studies)

Ángela Collados Aís (Universidad de Granada)

Helle Vrønning Dam (Aarhus Universitet)

Martin Djovčoš (Univerzita Mateja Bela)

Roch Duval (Université de Montréal)

Álvaro Echeverri (Université de Montréal)

Maureen Ehrensberger-Dow (Zürcher Fachhochschule)

Nermine El-Gebaly (Ain Shams University)

Fernando Ferreira Alves (Universidade do Minho)

Thierry Fontenelle (Centre de traduction des organes de l'Union européenne)

Clara Foz (Université d'Ottawa)

Lynne Franjié (Université Stendhal - Grenoble 3)

Nicolas Froeliger (Université Paris 7 - Diderot)

Chantal Gagnon (Université de Montréal)

Anna Ghiglione (Université de Montréal)

Dinda Gorlée (Universitetet i Bergen)

Jean-Marc Gouanvic (Concordia University)

Maity Guerreiro Siqueira (Universidade Federal do Rio Grande do Sul)

Bertha Gutiérrez Rodilla (Universidad de Salamanca)

Marie-Noëlle Guillot (University of East Anglia)

Eva Havu (Helsingin yliopisto)

Brenda Hosington (University of Warwick) 
Judith Inggs (University of the Witwatersrand Johannesburg)

Arnt Lykke Jakobsen (Handelshøjskolen i København)

Laurence Jay-Rayon

Klaus Kaindl (Universität Wien)

Dorothy Kelly (Universidad de Granada)

Don Kiraly (Johannes-Gutenberg-Universität Mainz)

Benoît Kremer (Association Internationale des Interprètes de Conférence)

Jan-Louis Kruger (Noordwes-Universiteit)

Francisco Lafarga (Universitat de Barcelona)

Christopher Larkosh (University of Massachusetts Darthmouth)

Élisabeth Le (University of Alberta)

Mu Lei (Guangdong University of Foreign Studies)

Defeng Li (University of London)

Wendan Li (University of North Carolina at Chapel Hill)

Kazem Lotfipour-Saedi (Université d'Ottawa)

Brigid Maher (La Trobe University)

Elizabeth Marshman (Université d'Ottawa)

Raquel Merino Álvarez (Universidad del País Vasco)

Denise Merkle (Université de Moncton)

Reine Meylaerts (Katholieke Universiteit Leuven)

Esther Monzó Nebot (Universitat Jaume I)

Robert Neather (Hong Kong Baptist University)

Alexis Nouss (Cardiff University)

Maeve Olohan (The University of Manchester)

Mariana Orozco Jutorán (Universitat Autònoma de Barcelona)

Réal Paquette (Université de Montréal)

Ilaria Parini (Università degli Studi di Milano)

Maria Pavesi (Università degli Studi di Pavia)

Luis Pegenaute Rodríguez (Universitat Pompeu Fabra)

Sandrine Peraldi (Institut Supérieur d'Interprétation et de Traduction)

Franz Pöchhacker (Universität Wien)

Fernando Prieto Ramos (Université de Genève)

Anthony Pym (Universitat Rovira i Virgili)

Serge Quérin (Université de Montréal)

Mariana Raffo (Université de Montréal)

Aline Rémaël (Artesis Hogeschool)

Margaret Rogers (University of Surrey)

Heidi Salaets (Katholieke Universiteit Leuven)

Christina Schäffner (Aston University)

Adriana Şerban (Université Paul-Valéry Montpellier 3)

Ascensión Sierra Soriano (Universitat d'Alacant)

Christopher Stone (Gallaudet University)

Madeleine Stratford (Université du Québec en Outaouais)

Anne Marie Taravella (Université de Sherbrooke)

Ira Torresi (Università di Bologna)

Daniel Toudic (Université Rennes 2) 
Marc Van Campenhoudt (Institut Supérieur de Traducteurs et Interprètes - Haute École de Bruxelles)

Cecilia Wadensjö (Stockholms universitet)

Malcolm Williams (Université d'Ottawa)

Marion Winters (Heriot-Watt University)

Zhijie Wu (Nanjing University of Science and Technology)

Patrick Zabalbeascoa (Universitat Pompeu Fabra)

Danielle Zaslavsky Rabinovici (El Colegio de México) 